\title{
TMExplorer: A Tumour Microenvironment Single-cell RNAseq Database and Search Tool
}

\author{
Erik Christensen ${ }^{1,2, *}$, Alaine Naidas ${ }^{2,3, *}$, Mia Husic $^{4}$, Parisa Shooshtari ${ }^{1,2,3,5,6}$
}

${ }^{1}$ Department of Computer Science, University of Western Ontario, London, ON, Canada

${ }^{2}$ Children's Health Research Institute, London Health Sciences Centre, London, ON, Canada

${ }^{3}$ Department of Pathology and Lab Medicine, University of Western Ontario, London, ON, Canada

${ }^{4}$ Hospital for Sick Children, Toronto, ON, Canada

${ }^{5}$ Ontario Institute for Cancer Research, Toronto, ON, Canada

${ }^{6}$ Correspondence to PS, Email: pshoosh@uwo.ca

* These authors contributed equally to this study.

\section{ABSTRACT}

Tumour microenvironments (TME) contain a variety of cells including but not limited to stromal fibroblasts, endothelial cells, immune cells, malignant cells, and cells of the tissues of origin, whose interactions likely influence tumour behaviour and response to cancer treatment. The specific composition of the TME can be elucidated using single-cell RNA sequencing (scRNA-seq) by measuring expression profiles of individual cells. Several scRNA-seq datasets from multiple cancer types have been published in recent years, yet we still lack a comprehensive database for the collection and presentation of TME data from these studies in an easily accessible format. We have thus built a database of TME scRNA-seq data, containing 21 TME scRNA-seq datasets from 12 different cancer types. We have also created an R package called TMExplorer, which provides an interface to easily search and access all available datasets and their metadata. Data and metadata are kept in a consistent format across all datasets, with multiple expression formats available depending on the use case. Users can view a table of metadata and select individual datasets or filter them by specific characteristics. Users may also select a specific type of cancer and view all published scRNA-seq data for that cancer type available in our database. Users are provided with an option to save the data in multiple formats in order to view or process it outside of R. Thus, the TMExplorer database 
and search tool allows for thorough examination of the TME using scRNA-seq in a way that is streamlined and allows for easy integration into already existing scRNA-seq analysis pipelines.

\section{INTRODUCTION}

Single-cell RNA sequencing (scRNA-seq) is a new technology that has emerged as an important tool to measure gene expression for individual cells, enabling the examination of cellular heterogeneity and tissue composition with incredible precision. This has been particularly applicable in cancer research for the study of tumour composition, heterogeneity and phenotype, all of which are directly impacted by the tumour microenivronment (TME). TMEs are composed of different stromal and cancer cell types whose interactions likely dictate different aspects of tumour behaviour, such as metastasis (1-4). Combined with scRNA-seq analysis methods, scRNA-seq enables us to dissect the TME into individual cells and investigate the different cell subpopulations that exist. Such investigations into the TME are becoming increasingly important, as tumour composition and heterogeneity can influence cancer progression and the outcome of cancer therapy (1,4-9).

With the advancement of scRNA-seq in cancer research, the number of TME datasets that are generated continues to increase, yet they can be difficult to access. Raw sequence reads generated by scRNAseq can be shared through online archives, such as the Sequence Read Archive (SRA) (10), however they exist as large files that require further processing to be analyzed, making data access a challenge. Already processed scRNA-seq data containing gene expression information can be accessed through online archives, such as the Genome Expression Omnibus (GEO) (11), and can be more easily downloaded for use in one's own analysis. Furthermore, to manage the growing abundance of publicly available scRNA-seq data, proper quality control and curation of datasets must be done $(12,13)$. Currently, several online databases offer curated collections of public scRNA-seq datasets, such as PanglaoDB (12), scRNASeqDB (13), JingleBells (14) and the Single Cell Portal created by the Broad Institute of MIT and Harvard (https://singlecell.broadinstitute.org/single_cell) (15). Most existing scRNA-seq databases include a mixture of samples from normal tissues and tissues affected by cancer or other diseases (12-14), while others focus primarily on samples from normal tissues $(16,17)$. A recently published toolkit called CReSCENT (18) contains only cancer scRNA-seq data, however it mainly acts as a cancer data analysis pipeline rather than a database. A comprehensive database for the collection and sharing of TME scRNA-seq datasets 
from a range of tumour types does not yet exist, and researchers interested in using publicly available TME data must search through several databases to collect relevant datasets for their study. A database of TME scRNA-seq samples will thus streamline the data collection steps required for researching cancer at a single-cell level, lowering the barrier for entry to this type of study.

It is likewise important that scRNA-seq databases are designed to facilitate streamlined data collection and analysis. This can include a search tool that allows users to select datasets based on desired characteristics. While existing databases include search tools, they provide few options in characteristics users are able to search for and often require users to browse through a metadata table prior to selecting datasets of interest. Furthermore, they are designed as webbased tools, and thus are not intended to be integrated into workflows (12-15). Workflow integration would enable users to access data directly in their pipelines, thus automating the data collection process and increasing analysis efficiency. A scRNA-seq database that is provided as an R-package and contains a comprehensive search tool which allows users to select datasets based on a wider variety of characteristics would make the data collection process easier for researchers.

Here, we present a curated collection of tumour scRNA-seq datasets made available as an Rpackage called TMExplorer. TMExplorer contains publicly available scRNA-seq datasets specific to TMEs from various tumour types collected from different scRNA-seq studies (13,5-9,19-31) and online databases (11,32). In addition to gene expression data, TMExplorer contains the corresponding cell type annotations and gene-signature information for several datasets, and provides a search tool enables users to search for multiple datasets according to 13 different characteristics (Table 1). When selecting datasets, users can review the metadata table first or they can retrieve datasets that match specific criteria without having to browse through the metadata table. While online databases require users to download a given dataset prior to use, TMExplorer allows users to access and search available datasets within R. Users can thus input the data directly into existing pipelines with only a few commands. Each dataset can be used directly within $\mathrm{R}$ as a SingleCellExperiment object, or exported as a gene expression matrix in multiple formats for use with other applications. Users interested in validating scRNA-seq analysis algorithms, as they apply to TME data, can easily access this information through TMExplorer and incorporate it into their pipelines. Altogether, 
TMExplorer makes it easier for researchers to access and share TME scRNA-seq datasets, facilitating the study of TMEs at the single-cell level in the field of cancer research.

\section{METHODS}

Data Collection: In order to collect the datasets, we searched the National Center for Biotechnology Information (NCBI) (33) for relevant scRNA-seq studies using the following keywords: single cell RNA sequencing, tumour, cancer, tumour microenvironment, and malignant. We then carefully reviewed the published literature and any associated data to confirm if they matched our criteria. Datasets were included in our data collection if they were publicly available as processed data, were generated by scRNA-seq and if they consisted of TME expression data. A total of 21 datasets originating from different types of human and mouse tumours were collected from online sources such as the NCBI's Gene Expression Omnibus (GEO) (11), ArrayExpress (32), and Github (34). Out of the 21 datasets we collected, 18 datasets originated from human tumours and 3 datasets originated from mouse tumours. Descriptions of the collected datasets are provided in Table 2. Metadata for each dataset, such as tumour type and number of cells sequenced, were collected from descriptions in the corresponding publications and/or from the online sources that the datasets were obtained from. If publicly available, we also retrieved cell-type annotations and/or gene signature information that accompanied the datasets.

Data Curation: Datasets found on GEO often contain extra information such as Ensemble ID or chromosome region in additional rows or columns. We modified all datasets using $\mathrm{R}$ to ensure they followed a similar genes-by-cells format with the gene column serving as an index. Having a similar format for datasets reduces the preprocessing required to use this data in other analysis pipelines.

There are three main components to each dataset in our database: gene-by-cell expression matrices, cell type labels, and gene signatures. The cell type labels are R dataframes with two columns; one contains every cell barcode present in the expression matrix, the other one contains that cell's type. The gene signatures are stored in R dataframes containing one column per cell type, with a list of genes that are differentially expressed by that cell type and reported in the original paper in which each dataset was first introduced. All data for each dataset is accessible within a single object in order to make it as easy to use as possible. 
Since R BioConductor has existing infrastructure for working with scRNA-seq data $(35,36)$, we used it as the platform to build our package upon. In order to maintain compatibility with existing Bioconductor software, we return all datasets as SingleCellExperiment objects (36). Figure 1 shows the structure of a SingleCellExperiment object, where the expression data is stored as a named assay, cell type labels (if present) are stored under colData(), and all other information is stored in a metadata list.

- Expression Data: Named assays allow certain formats to be easily accessed with getter functions such as counts() and tpm(), while other formats can still be accessed with the $\operatorname{assay}()$ getter function (36). All SingleCellExperiments have one assay named according to the type of score (e.g. Counts and TPM) represented in that object. Calling assay()_returns an expression matrix with rows of genes and columns of cells.

- Cell Type Labels: ColData stores metadata for the columns in the assay matrix. In our case this refers to the cell type annotations, if they are available. ColData is a dataframe that always has one row for every column in the assay matrix, ensuring that there is a label present for every cell. If the cell type is not available for a given cell, it is labelled as "unknown".

- Metadata: The metadata list serves to store any other information that does not fit into a pre-existing attribute of the SingleCellExperiment object, and is accessed with the metadata() function. This named list contains the signature gene sets, available score types, tumour and host organism type, sequencing technology, author, and all other descriptive information as strings. All information that is available in the metadata table can be accessed by calling the query function of TMExplorer (i.e. queryTME) with the metadata_only parameter set to true.

Metadata: After collecting the datasets, corresponding metadata was compiled into a table which serves as the core of the package. The metadata table contains information such as GEO accession, author, journal, year, PMID, sequencing technology, expression score type(s), source organism, type of cancer, number of patients, tumours, cells and genes, and the database that the data was obtained from. All items in the metadata table were chosen as either entities 
that distinguish one dataset from the others or criteria that may make a dataset or group of datasets interesting to researchers (e.g. a specific tumour type or availability of cell labels or gene signatures). Users can view the available data using the metadata table and decide which dataset best fits their needs.

Database Query: TMExplorer provides a query function (i.e. queryTME) that users can employ in order to select multiple datasets based on their desired characteristics. For example, users can select specific studies by PMID or GEO accession, or filter subsets by sequencing technology, whether cell type labels or cell type signature gene sets are available etc. Sequencing technology, score type, organism, tumour type, and year were all chosen as search parameters because they represent differences in the type of data and make it easier to find data that fits the needs of different studies. We have also made it possible to search for datasets for which the cell labels and gene signatures are available. This facilitates developing and testing algorithms that require specific types of dataset information. For example, testing cell classification algorithms requires cell labels that can be used as a gold standard, and many existing algorithms require gene signatures that represent the cell types in the dataset $(37,38)$.

Alternative Experiments: For several datasets, gene expressions are available in multiple score types including raw counts and normalized data by FPKM, TPM or CPM. In order to store each dataset in multiple score types, we used nested SingleCellExperiments objects with the alternative experiments (altExps) concept. Alternative experiments are guaranteed to have the same dimensions as the primary object, but can be kept separate for use in other pipelines (36). This allows users to download multiple types of scoring for use in different steps of analysis while still being able to access each dataset through a single object. Being able to download multiple score types allows our datasets to be used in a variety of algorithms that require a specific type of score, and keeping them separated as nested objects prevents accidentally applying an algorithm to the wrong score type.

Dense vs. Sparse Data Formats: In order to reduce the memory requirements for working with large datasets, expression data is optionally available as a sparse matrix. We implemented sparse matrices using the $d g C M a t r i x$ class from R Matrix (39). This reduces memory usage by only storing non-zero expression values. With sparse matrices, the memory required to store a dataset is reduced by as much as $8 \mathrm{~Gb}$ for a dataset with 51,775 cells and 22,533 genes. It should be noted that not all software packages are compatible with sparse matrices, and 
converting large datasets from sparse to dense may crash $\mathrm{R}$ on machines with low memory. Thus users should confirm that their algorithms support sparse matrices before using them. By default, TMExplorer returns dense matrices to avoid these problems.

Exporting Data in Multiple Formats: Several tools for scRNA-seq analysis are written in R and therefore a SingleCellExperiment object can easily be incorporated into these pipelines and tools. However, many other analysis tools are written in Python or as webapps $(18,40,41)$. To facilitate the use of TMExplorer with these tools, we wrote a function saveTME that writes individual TME datasets to disk as CSV or Matrix Market files, depending on whether data was loaded as dense or sparse matrices by queryTME, respectively. SaveTME takes a SingleCellExperiment object and a path to an output directory as parameters and saves the gene expression matrix, cell type labels, and cell type signature gene sets to disk. The resulting files can then be converted as needed and used in other applications.

\section{RESULTS}

Overview of the TMExplorer Package: To make it as easy as possible to integrate TMExplorer into other pipelines, all interactions with the package are done directly in R. Here, the queryTME function serves as the primary interface for the package, allowing users to view the metadata for all available datasets, or select a subset of datasets according to descriptive criteria (Figure 2a). queryTME provides a set of parameters (Table 1) used to select a subset of datasets according to characteristics. To review the available datasets, the metadata_only parameter should be set to TRUE when querying the package, and a table describing the datasets will be returned instead of the datasets themselves. The search parameters can be used to find relevant data without requiring users to review the metadata table first, lowering the barrier for use. For example, users looking for a certain type of cancer, such as melanoma, can search using queryTME(tumour_type="Melanoma") without needing to first examine the metadata for datasets contain melanoma cancers.

After querying the database, a list of SingleCellExperiment objects are returned. The objects in this list can then be passed to any other algorithms that accept a SingleCellExperiment object, sparse dgCMatrix, or dense gene expression matrix for inclusion in a pipeline (Figure 3). Alternatively, the saveTME function can be used to write the returned data to disk for further manipulation or use in applications outside of $\mathrm{R}$ (Figure $2 \mathrm{~b}$ ). Figure 3 shows how saveTME 
can be used to save data for analysis in Python. In order to maintain consistency, the returned value is always a list of results, whether or not multiple datasets match the query.

TMExplorer Database Contents: TMExplorer is a curated collection of TME scRNA-seq datasets that have been made available as an R-package. We created TMExplorer to improve accessibility and sharing of tumour scRNA-seq data. It acts as a single-entry point to various tumour scRNA-seq datasets for users interested in studying gene expression of the TME at the single-cell level. Currently, the collection contains 21 datasets, including 18 datasets derived from human tumours and 3 datasets derived from mouse tumours. This comprises 12 different cancer types including leukemia (22,24), breast $(2,27,28)$, colorectal $(3)$, glioblastoma $(7,9,31)$, glioma (23), head and neck (20), astrocytoma (21), oligodendroglioma (8), melanoma $(1,5,19)$, lung carcinoma (6), pancreatic $(25,29)$ and prostate $(26)$. Numbers of cells and genes vary across datasets and fall within the range of 5,796-57,915 genes and 74-57,530 cells. Each dataset is provided as processed gene expression data. We did not include raw scRNA-seq data (i.e. FASTQ files) in our collection because these files tend to be very large and can be accessed through the SRA, if available. Out of the 21 datasets, cell-type annotations are also provided for 12 datasets and gene signature information is provided for 9 datasets, so that users may access and use this information in their analyses. Users can browse through the available datasets using the metadata table and then choose which dataset(s) they would like to analyze. Users can also save the datasets for use outside of R, for instance in Python or web-based analysis pipelines.

TMExplorer Search Capability: An important feature of TMExplorer is that it acts as both a database and search tool that can be easily implemented in one's own workflow. Some other currently available scRNA-seq databases have a search function, but cannot be easily integrated into workflows because they are web-based (12-15). Currently available R-based scRNA-seq databases lack built in search tools, requiring users to access vignettes to see the available data before it can be retrieved for use in a pipeline (16). TMExplorer provides a search tool that allows users to search for datasets that fit their needs by tumour type, sequencing technology, source organism, and more (Table 1), all from the R command line. This makes TMExplorer an improvement over both R-based and web-based databases because users are able to browse and query data from the same console they are using for analysis. By including a search tool and database in a single package, TMExplorer provides a single point of entry to include TME scRNA-seq data into data analysis pipelines. 
Case Studies: In this section, we bring two example applications where TMExplorer can be used to facilitate data analysis. In the case study 1, we show how TMExplorer can be combined with automated cell-type identification algorithms to identify different cell types in TME scRNA-seq data. Here, we also show how users can return datasets with both the signature gene sets and gold standard annotations needed for testing cell-type identification. In case study 2, we show how TMExplorer can be integrated with the algorithms for inferencing copynumber variations in individual cells and facilitate the separation of malignant and nonmalignant cells in multiple tumour scRNA-seq datasets of the same cancer type.

\section{Case Study 1: Identifving different cell types in TME ScRNA-seq data}

Often, when using TME scRNA-seq data, we are interested in the cellular composition of the dataset. In order to find this, automated cell type identification algorithms are used. This is usually done by first clustering the cells, and then assigning appropriate cell type labels to each cluster (42). In Figure 4, we show how TMExplorer can be combined with a clustering method (e.g. Seurat (43)) and a cluster labelling method (e.g. GSVA (38)) to create a workflow for the identification of cell populations within a dataset. Seurat requires only the gene expression matrix to perform clustering, but GSVA requires a list of cell-type signature gene sets in addition to the expression matrix. TMExplorer can return all of the datasets that have signature gene sets available using queryTME(has_signatures $=T R U E)$. If after identifying the cell types within a dataset, users want to assess the performance of their workflow by comparing the automated annotations to those reported alongside the dataset, the has_truth $=$ TRUE parameter can be added to queryTME to only return datasets that have gold standard labels available. Seurat and GSVA can be replaced by any other tool that accepts a SingleCellExperiment object or a matrix of gene expression values, providing flexibility for users to incorporate TMExplorer into their own workflows.

\section{Case Study 2: Inferencing copy number variations in multiple datasets of the same cancer}

type. Single cell sequencing is an important tool that enables the dissection of TMEs into malignant and non-malignant cells. Researchers interested in comparing the tumour composition across different datasets of a specific cancer type would have to collect datasets from different sources prior to application of separation methods. With TMExplorer, users can easily access multiple datasets of a specific tumour type, as well as the accompanying cell type annotations and/or gene signature information from one location, thus avoiding inconsistencies 
when acquiring data from different databases. TMExplorer can be easily incorporated with other packages into workflows for the analysis of scRNA-seq data, therefore enabling users to access and use the data entirely within $\mathrm{R}$.

Figure 5 displays an example workflow that uses queryTME(tumour_type = "Glioblastoma") to retrieve datasets of a specific cancer type (i.e. glioblastoma) for use in the downstream analysis. In this example, we retrieved glioblastoma datasets from the TMExplorer database as SingleCellExperiment objects and converted them to gene expression count data matrices. We then applied a copy number variation (CNV) inferencing method called CONICSmat (44) to each of the datasets individually, and generated heatmaps displaying the inferred CNV patterns. This allowed us to separate malignant and non-malignant cells considering their long-range $\mathrm{CNV}$ patterns. The proportion of malignant and non-malignant cells and the patterns of CNV across the different datasets can then be compared.

\section{DISCUSSION}

The emergence of single-cell RNA sequencing has enabled the study of tumour composition and phenotype. With the increasing use of scRNA-seq in cancer research, scRNA-seq data from TMEs continues to be generated and published. In order to streamline the data collection process for researchers interested in studying the TME, we created a curated database of TME scRNA-seq datasets, made available as an R-package called TMExplorer. Here we have built a database using a variety of cancers from multiple sources. We searched NCBI $(11,33)$ for TME scRNA-seq datasets that contain gene expression data, as well as comprehensive metadata such as tumour type, sequencing technology, cell type annotations, and gene signatures. In total, 21 datasets representing 12 different cancers are represented, along with their cell type annotations and cell type signature gene sets if they were available.

TMExplorer addresses a gap in currently available scRNA-seq databases by providing a focused, easily accessible database as an R package. TMExplorer has several advantages over other currently available scRNA-seq databases, the most prominent being:

1. Existing curated scRNA-seq databases consist of mostly normal tissue or non-cancer data and relatively few cancer datasets. To allow researchers to easily locate and access TME scRNA-seq data, we curated publicly available TME datasets and made them 
available in a database accessible as an R package. With TMExplorer, researchers can access all publicly available TME scRNA-seq datasets from a single location and can also return multiple datasets that match their desired criteria with a single command.

2. TMExplorer provides a variety of search parameters (Table 1) that can be used to return a subset of the available data that matches specific criteria. The parameters were designed so that users can search for matching datasets without having to first view a list of all available datasets, making it easier and faster to access data of interest.

3. The majority of existing scRNA-seq databases can only be accessed online as webbased tools and are not easily incorporated into pipelines for analysis of scRNA-seq data. Since many researchers use R or Python for their analyses, we chose to provide TMExplorer as an R-package so that it may be easily integrated into existing pipelines.

4. Some analyses require more than just gene expression information, and TMExplorer provides cell type annotations and cell type signature gene sets alongside gene expression matrices, where they are available. This facilitates the use of a wider range of analysis methods without requiring additional work from the researchers.

We plan to keep the package updated with new datasets as they are published. Users of the package doing their own novel research will have access to an issue template on Github where they can submit their data for inclusion. The interested users will need to provide their scRNAseq data as raw counts or normalized data and the corresponding metadata. Since the package is open source, those same users can create a fork of the repository and build it from source with their own data for pre-publication work.

In summary, TMExplorer allows researchers to easily access, share and integrate TME scRNAseq data into their own analysis pipelines. TMExplorer can be used to access data needed for the validation of new algorithms and to allow researchers interested in the tumour microenvironment to study specific types of cancer.

\section{SOFTWARE AND DATA AVAILABILITY}

All included datasets are from free, public sources $(11,33)$ and all source code is freely available on Github (https://github.com/shooshtarilab/TMExplorer). Additionally, it is currently undergoing review for acceptance to BioConductor. Users wishing to have their data 
bioRxiv preprint doi: https://doi.org/10.1101/2020.10.31.362988; this version posted November 2,2020 . The copyright holder for this preprint (which was not certified by peer review) is the author/funder, who has granted bioRxiv a license to display the preprint in perpetuity. It is made available under aCC-BY-NC-ND 4.0 International license.

added to TMExplorer can open an issue at https://github.com/shooshtarilab/TMExplorer/issues with a link to their study and data along with a brief description and we will review it for inclusion.

\section{ACKNOWLEDGEMENTS}

This work was supported by the research grants from Lawson Health Research Institute, the University of Western Ontario, and Ontario Institute for Cancer Research.

\section{CONFLICTS OF INTEREST}

None.

\section{REFERENCES}

1. Tirosh, I., Izar, B., Prakadan, S. M., et al. (2016) Dissecting the multicellular ecosystem of metastatic melanoma by single-cell RNA-seq. Science.

2. Chung, W., Eum, H. H., Lee, H. O., et al. (2017) Single-cell RNA-seq enables comprehensive tumour and immune cell profiling in primary breast cancer. Nat. Commun.

3. Li, H., Courtois, E. T., Sengupta, D., et al. (2017) Reference component analysis of single-cell transcriptomes elucidates cellular heterogeneity in human colorectal tumors. Nat. Genet.

4. Tirosh, I. and Suvà, M. L. (2019) Deciphering Human Tumor Biology by Single-Cell Expression Profiling. Annual Review of Cancer Biology.

5. Jerby-Arnon, L., Shah, P., Cuoco, M. S., et al. (2018) A Cancer Cell Program Promotes T Cell Exclusion and Resistance to Checkpoint Blockade. Cell.

6. Lambrechts, D., Wauters, E., Boeckx, B., et al. (2018) Phenotype molding of stromal cells in the lung tumor microenvironment. Nat. Med.

7. Darmanis, S., Sloan, S. A., Croote, D., et al. (2017) Single-Cell RNA-Seq Analysis of Infiltrating Neoplastic Cells at the Migrating Front of Human Glioblastoma. Cell Rep.

8. Tirosh, I., Venteicher, A. S., Hebert, C., et al. (2016) Single-cell RNA-seq supports a developmental hierarchy in human oligodendroglioma. Nature.

9. Patel, A. P., Tirosh, I., Trombetta, J. J., et al. (2014) Single-cell RNA-seq highlights intratumoral heterogeneity in primary glioblastoma. Science.

10. Shumway, M., Cochrane, G. and Sugawara, H. (2010) Archiving next generation sequencing data. Nucleic Acids Res., 38, D870-1.

11. Edgar, R., Domrachev, M. and Lash, A. E. (2002) Gene Expression Omnibus: NCBI gene expression and hybridization array data repository. Nucleic Acids Res., 30, $207-$ 210.

12. Franzén, O., Gan, L. M. and Björkegren, J. L. M. (2019) PanglaoDB: A web server for exploration of mouse and human single-cell RNA sequencing data. Database.

13. Cao, Y., Zhu, J., Han, G., et al. (2017) scRNASeqDB: a database for gene expression profiling in human single cell by RNA-seq. bioRxiv.

14. Ner-Gaon, H., Melchior, A., Golan, N., et al. (2017) JingleBells: A Repository of 
Immune-Related Single-Cell RNA-Sequencing Datasets. The Journal of Immunology.

15. The Broad Institute of MIT and Harvard (2019) Single Cell Portal. Single Cell Portal https://singlecell.broadinstitute.org/single_cell (accessed Aug 20, 2020).

16. Risso, D. and Cole, M. (2016) scRNAseq: A collection of public single-cell RNA-seq datasets. $R$ package version, 1.

17. Wang, Z., Feng, X. and Li, S. C. (2019) SCDevDB: A database for insights into singlecell gene expression profiles during human developmental processes. Front. Genet.

18. Mohanraj, S., Díaz-Mejía, J. J., Pham, M. D., et al. (2020) CReSCENT: CanceR Single Cell ExpressioN Toolkit. Nucleic Acids Res.

19. Davidson, S., Efremova, M., Riedel, A., et al. (2018) Single-cell RNA sequencing reveals a dynamic stromal niche within the evolving tumour microenvironment. bioRxiv.

20. Puram, S. V., Tirosh, I., Parikh, A. S., et al. (2017) Single-Cell Transcriptomic Analysis of Primary and Metastatic Tumor Ecosystems in Head and Neck Cancer. Cell.

21. Venteicher, A. S., Tirosh, I., Hebert, C., et al. (2017) Decoupling genetics, lineages, and microenvironment in IDH-mutant gliomas by single-cell RNA-seq. Science.

22. Giustacchini, A., Thongjuea, S., Barkas, N., et al. (2017) Single-cell transcriptomics uncovers distinct molecular signatures of stem cells in chronic myeloid leukemia. Nat. Med.

23. Filbin, M. G., Tirosh, I., Hovestadt, V., et al. (2018) Developmental and oncogenic programs in H3K27M gliomas dissected by single-cell RNA-seq. Science.

24. van Galen, P., Hovestadt, V., Wadsworth, M. H., et al. (2019) Single-Cell RNA-Seq Reveals AML Hierarchies Relevant to Disease Progression and Immunity. Cell.

25. Ting, D. T., Wittner, B. S., Ligorio, M., et al. (2014) Single-cell RNA sequencing identifies extracellular matrix gene expression by pancreatic circulating tumor cells. Cell Rep.

26. Miyamoto, D. T., Zheng, Y., Wittner, B. S., et al. (2015) RNA-Seq of single prostate CTCs implicates noncanonical Wnt signaling in antiandrogen resistance. Science.

27. Jordan, N. V., Bardia, A., Wittner, B. S., et al. (2016) HER2 expression identifies dynamic functional states within circulating breast cancer cells. Nature.

28. Azizi, E., Carr, A. J., Plitas, G., et al. (2018) Single-Cell Map of Diverse Immune Phenotypes in the Breast Tumor Microenvironment. Cell.

29. Peng, J., Sun, B. F., Chen, C. Y., et al. (2019) Single-cell RNA-seq highlights intratumoral heterogeneity and malignant progression in pancreatic ductal adenocarcinoma. Cell Res.

30. Kumar, M. P., Du, J., Lagoudas, G., et al. (2018) Analysis of Single-Cell RNA-Seq Identifies Cell-Cell Communication Associated with Tumor Characteristics. Cell Rep.

31. Zhao, Y., Carter, R., Natarajan, S., et al. (2019) Single-cell RNA sequencing reveals the impact of chromosomal instability on glioblastoma cancer stem cells. BMC Med. Genomics.

32. Athar, A., Füllgrabe, A., George, N., et al. (2019) ArrayExpress update - From bulk to single-cell expression data. Nucleic Acids Res.

33. (1988) National Center for Biotechnology Information (NCBI). National Center for Biotechnology Information (NCBI) https://www.ncbi.nlm.nih.gov/ (accessed Aug 20, 2020).

34. GitHub, I. (2007) GitHub. GitHub https://github.com/ (accessed Aug 21, 2020).

35. Huber, W., Carey, V. J., Gentleman, R., et al. (2015) Orchestrating high-throughput genomic analysis with Bioconductor. Nat. Methods, 12, 115-121.

36. Lun, A., Risso, D. and Korthauer, K. (2019) SingleCellExperiment: S4 classes for single cell data. $R$ package version, 1.

37. Newman, A. M., Liu, C. L., Green, M. R., et al. (2015) Robust enumeration of cell 
subsets from tissue expression profiles. Nat. Methods, 12, 453-457.

38. Hänzelmann, S., Castelo, R. and Guinney, J. (2013) GSVA: gene set variation analysis for microarray and RNA-seq data. BMC Bioinformatics, $14,7$.

39. Maechler, M. (2019) Matrix: Sparse and Dense Matrix Classes and Methods. R package version 1.2-18. Matrix: Sparse and Dense Matrix Classes and Methods. R package version 1.2-18 (2019) .

40. Franzén, O. and Björkegren, J. L. M. (2020) alona: a web server for single-cell RNA-seq analysis. Bioinformatics, 36, 3910-3912.

41. Afgan, E., Baker, D., Batut, B., et al. (2018) The Galaxy platform for accessible, reproducible and collaborative biomedical analyses: 2018 update. Nucleic Acids Res., 46, W537-W544.

42. Diaz-Mejia, J. J., Meng, E. C., Pico, A. R., et al. (2019) Evaluation of methods to assign cell type labels to cell clusters from single-cell RNA-sequencing data. F1000Res., 8.

43. Stuart, T., Butler, A., Hoffman, P., et al. (2019) Comprehensive Integration of SingleCell Data. Cell, 177, 1888-1902.e21.

44. Müller, S., Cho, A., Liu, S. J., et al. (2018) CONICS integrates scRNA-seq with DNA sequencing to map gene expression to tumor sub-clones. Bioinformatics, 34, 3217-3219. 


\section{FIGURES}

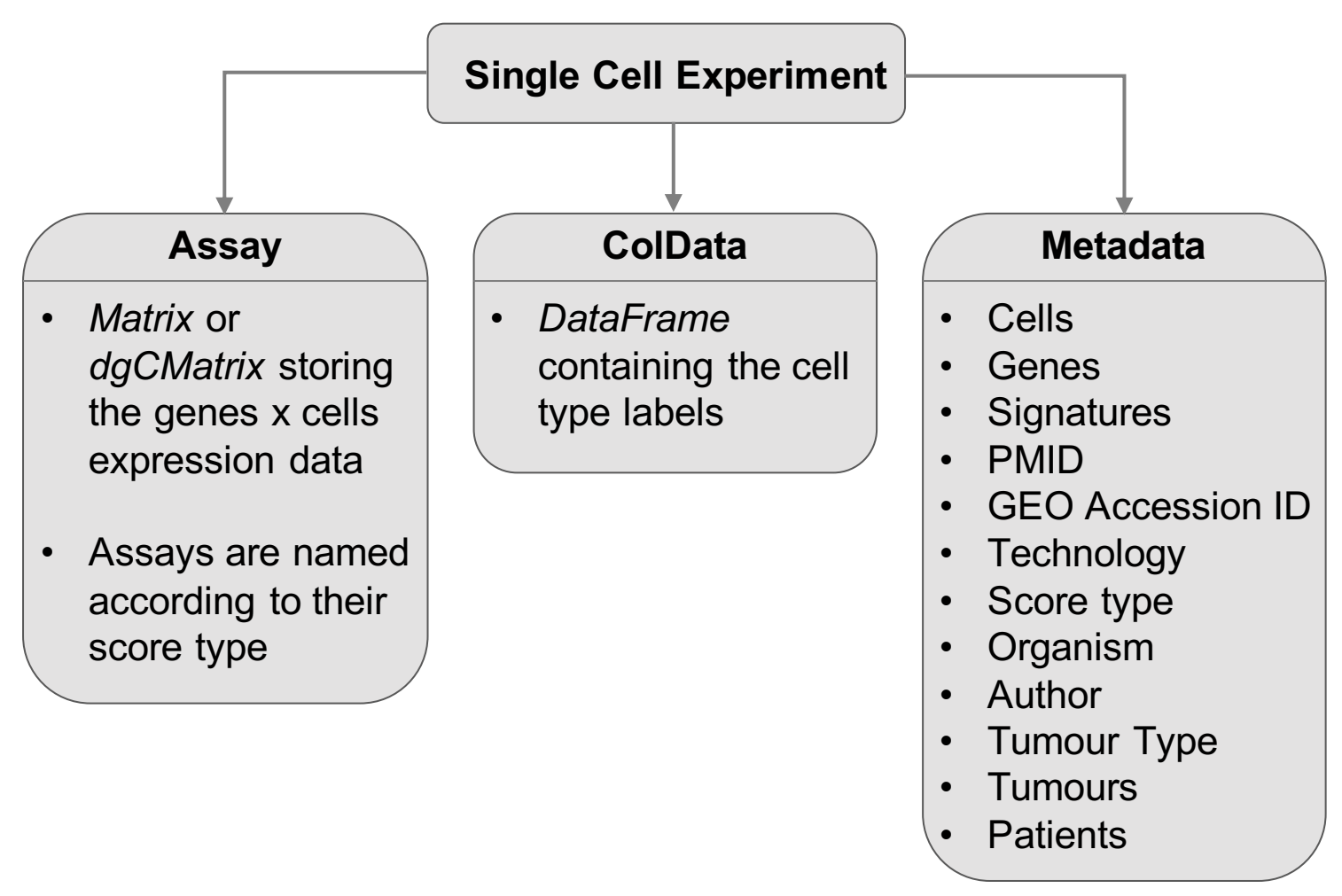

Figure 1: The format of the SingleCellExperiment objects containing TME datasets. The Assay is a matrix or dgCMatrix containing the gene expression table, named according to the type of score (i.e. an Assay containing raw counts would be named "Counts"); colData is a DataFrame with the number of rows equal to the number of columns in the Assay and describes the cells in the dataset; Metadata is a named list of additional metadata objects describing the dataset. A SingleCellExperiment object may contain one or more AltExps, which are nested SingleCellExperiment objects containing a different score type in the Assay. 
A

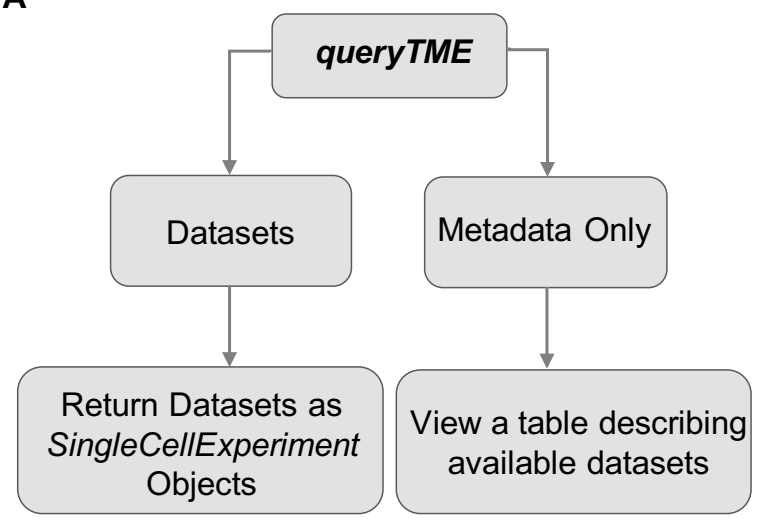

B

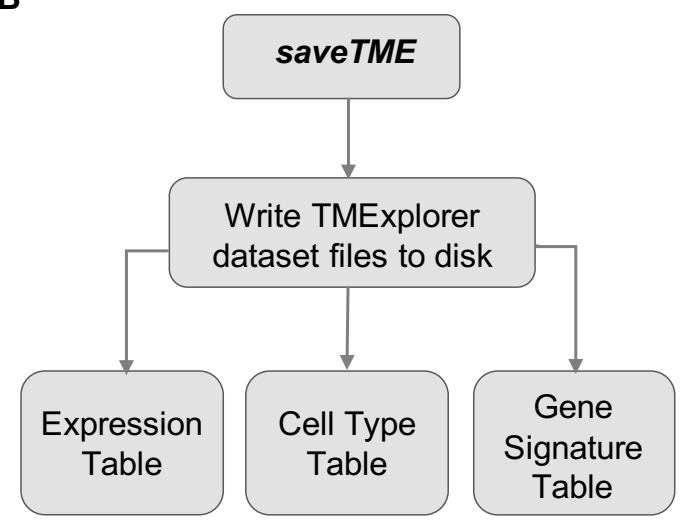

Figure 2: An overview of the main functions of TMExplorer. A) queryTME allows users to search and return datasets in either a descriptive table or as a list of SingleCellExperiment objects for analysis. B) saveTME allows users to write datasets to disk. For each dataset written to disk, up to three files are created; a table storing the expression data as either a CSV or matrix market file, depending on whether a dense or sparse matrix is passed to the function; a table containing the cells and their truth label, if available; and a table containing the cell type signature gene sets, if available. 


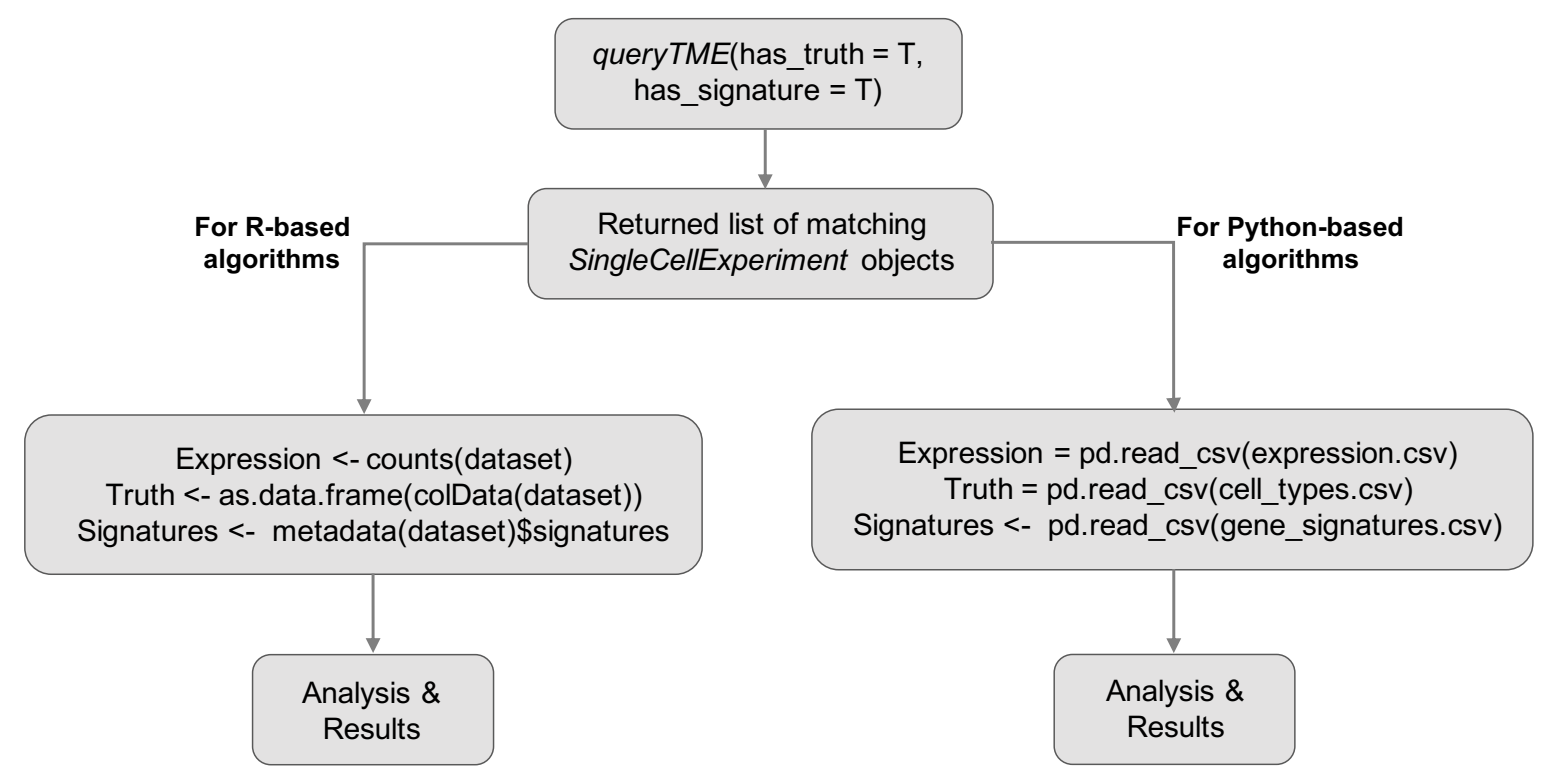

Figure 3: An example workflow of using TMExplorer to obtain datasets for the downstream analysis using Python and R. Users start by using queryTME to return all datasets that have cell type labels and cell type signature gene sets, which will get a list of matching datasets contained in SingleCellExperiment objects. Then, for R based algorithms, users can pass the SingleCellExperiments directly if that is supported, or users can pass the individual components required. For Python based algorithms, saveTME can be used to save the files for each dataset to disk, which can then be opened in Python for analysis. 


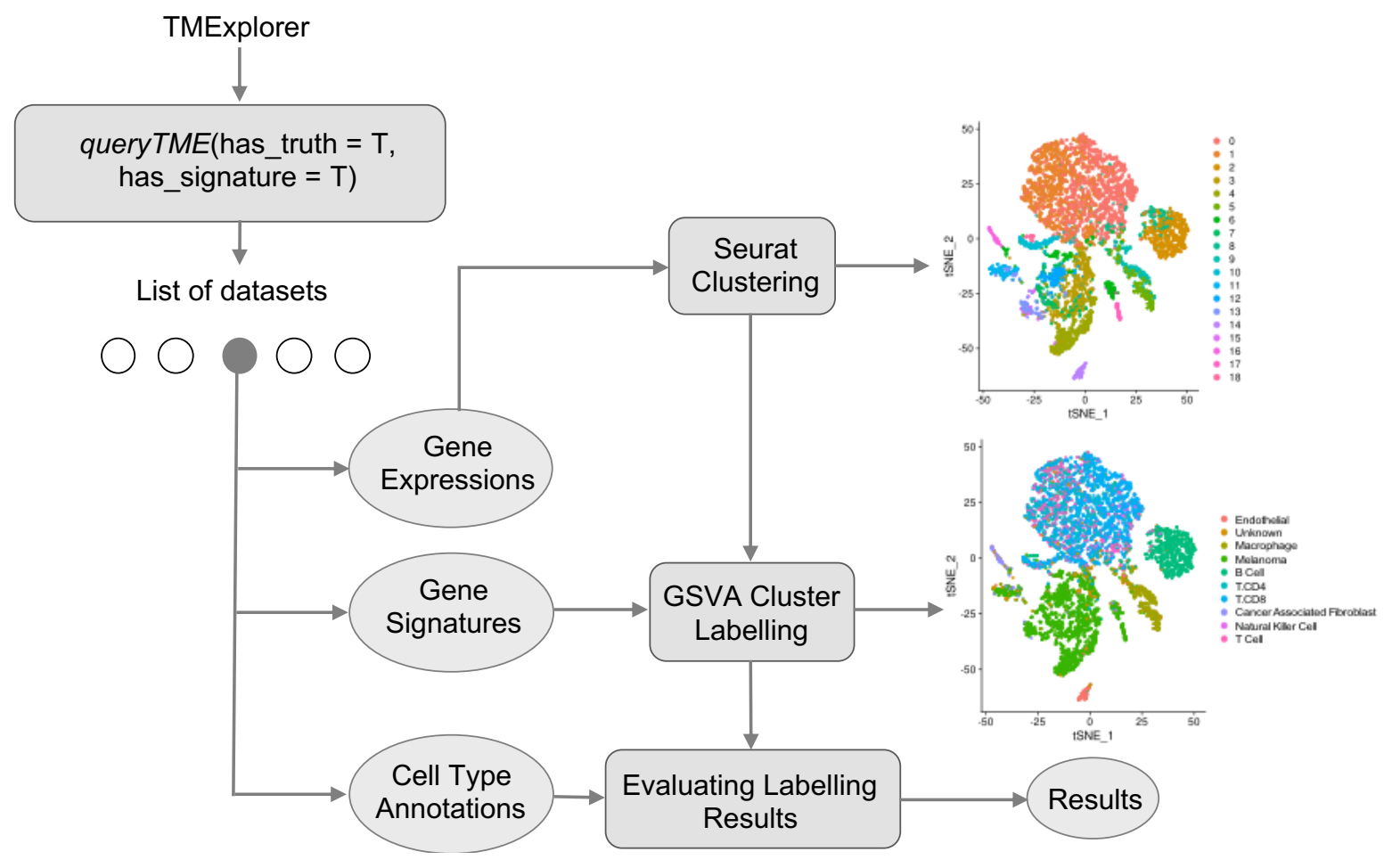

Figure 4: A case study on using TMExplorer to identify cell types. A case study showing how TMExplorer can be used to obtain datasets that can be used for cell cluster labelling via Seurat and GSVA. queryTME can be used to return those datasets which have both gene signatures and cell type annotations required for testing the automated identification of cell types. The expression data can be passed to Seurat for cell clustering, and the gene signatures can be used by GSVA to identify the cell types in Seurat's clusters. Finally, the cell type annotations can be used as the truth labels to measure the performance of the results obtained by Seurat clustering followed by GSVA. 
bioRxiv preprint doi: https://doi.org/10.1101/2020.10.31.362988; this version posted November 2,2020 . The copyright holder for this preprint (which was not certified by peer review) is the author/funder, who has granted bioRxiv a license to display the preprint in perpetuity. It is made available under aCC-BY-NC-ND 4.0 International license.

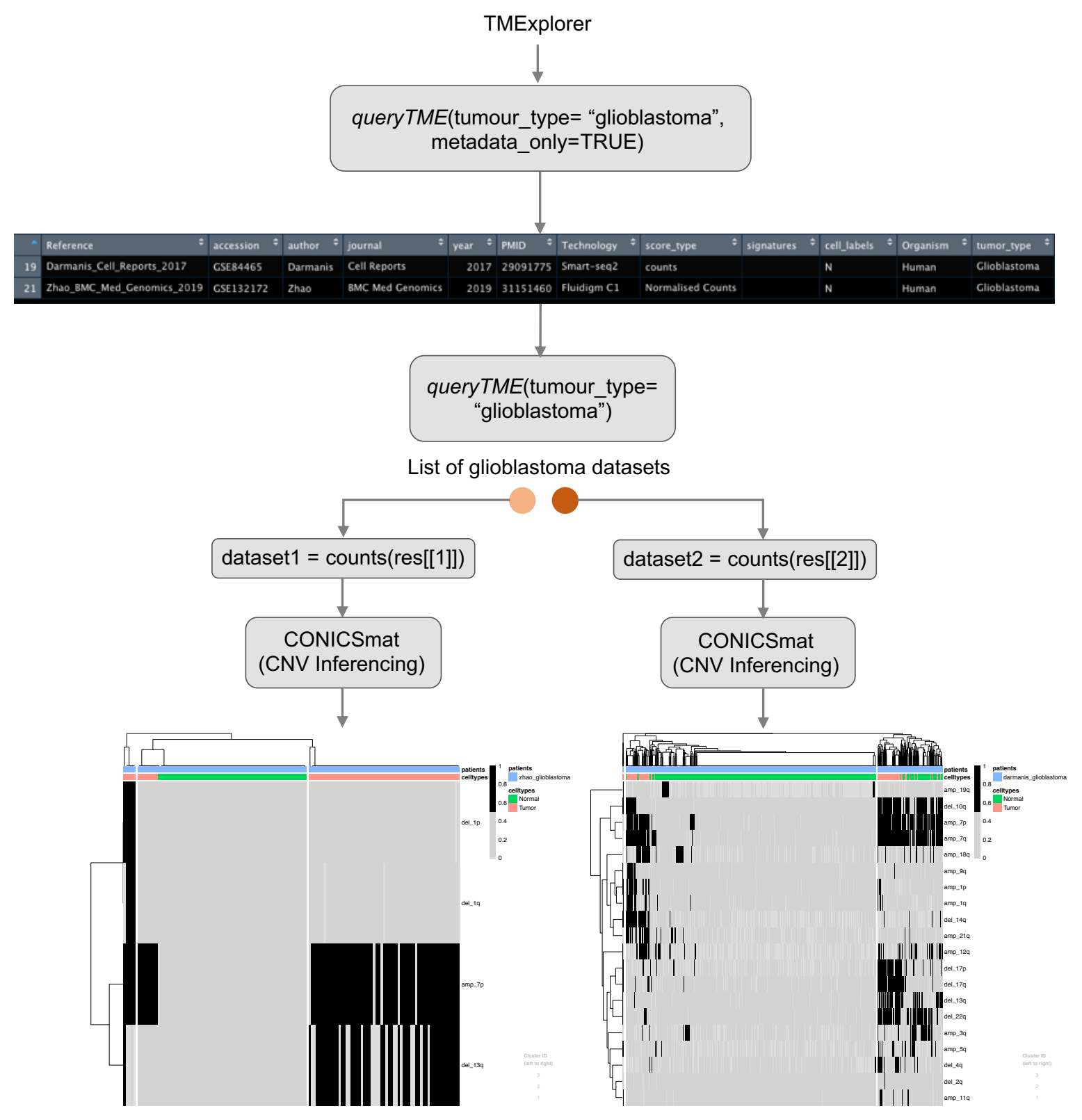

Figure 5: A case study on using TMExplorer for inferring CNVs. A case study showing how TMExplorer can be used to obtain multiple datasets for a specific tumour type, to be used with CNV-based separation methods, such as CONICSmat. QueryTME returns datasets of a specific tumour type, such as Glioblastoma. These datasets can then be inputted directly into large-scale CNV inferencing methods, such as CONICSmat. 
Table 1: A list of search parameters that can be passed to queryTME in order to filter the available datasets.

\begin{tabular}{|l|l|}
\hline Search Parameter & Description \\
\hline geo_accession & Search by GEO accession \\
\hline score_type & Search by type of score available \\
\hline has_signatures & $\begin{array}{l}\text { Search by presence of cell type signature } \\
\text { gene sets }\end{array}$ \\
\hline has_truth & Search by presence of cell type annotations \\
\hline tumour_type & Search by type of tumour \\
\hline author & Search by first author \\
\hline journal & Search by publication journal \\
\hline year & Search by publication year \\
\hline pmid & Search by PMID \\
\hline sequence_tech & Search by sequencing technology \\
\hline organism & Specify a list of score formats to download. \\
\hline sparse & Additional formats will be stored in altExps \\
\hline download_format & \\
\hline
\end{tabular}


Table 2: List of tumor microenvironment scRNA-seq datasets included in TMExplorer

\begin{tabular}{|c|c|c|c|c|c|c|c|}
\hline Dataset & Cancer type & $\begin{array}{l}\text { Sequencing } \\
\text { Technology }\end{array}$ & Number of tumors & $\begin{array}{l}\text { Number } \\
\text { of cells }\end{array}$ & $\begin{array}{l}\text { Number } \\
\text { of genes }\end{array}$ & $\begin{array}{l}\text { Annotation } \\
\text { available? }\end{array}$ & $\begin{array}{l}\text { Gene } \\
\text { signature } \\
\text { available? }\end{array}$ \\
\hline Patel et al. Science 2014 & $\begin{array}{l}\text { Primary } \\
\text { Glioblastoma }\end{array}$ & SMART-seq & $\begin{array}{l}5 \text { human } \\
\text { primary glioblastoma } \\
\text { tumors }\end{array}$ & 1,456 & 5,796 & Yes & No \\
\hline Tirosh et al. Science 2016 & $\begin{array}{l}\text { Metastatic } \\
\text { melanoma }\end{array}$ & SMART-seq2 & $\begin{array}{l}19 \text { human melanoma } \\
\text { tumors }\end{array}$ & 4,645 & 23,686 & Yes & Yes \\
\hline Tirosh et al. Nature 2016 & Oligodendroglioma & SMART-seq2 & $\begin{array}{l}6 \text { human } \\
\text { oligodendroglioma } \\
\text { tumors }\end{array}$ & 4,347 & 23,686 & Yes & Yes \\
\hline $\begin{array}{l}\text { Venteicher et al. Science } \\
2017\end{array}$ & $\begin{array}{l}\text { IDH-mutant } \\
\text { astrocytoma }\end{array}$ & SMART-seq2 & $\begin{array}{l}10 \text { human } \\
\text { IDH-mutant } \\
\text { Astrocytoma } \\
\text { tumors }\end{array}$ & 6,341 & 23,686 & No & No \\
\hline $\begin{array}{l}\text { Li et al. Nature Genetics } \\
2017\end{array}$ & Colorectal cancer & Fluidigm C1 & $\begin{array}{l}11 \text { human primary } \\
\text { colorectal cancer } \\
\text { tumors }\end{array}$ & 376 & 57,241 & Yes & Yes \\
\hline $\begin{array}{l}\text { Chung et al. Nature } \\
\text { Commun } 2017\end{array}$ & $\begin{array}{l}\text { Primary breast } \\
\text { cancer }\end{array}$ & Fluidigm C1 & $\begin{array}{l}11 \text { human primary } \\
\text { breast cancer tumors }\end{array}$ & 564 & 57,915 & Yes & Yes \\
\hline Puram et al. Cell 2017 & $\begin{array}{l}\text { Head and neck } \\
\text { squamous cell } \\
\text { carcinoma }\end{array}$ & SMART-seq2 & $\begin{array}{l}18 \text { human primary } \\
\text { oral cavity tumors and } \\
5 \text { lymph node } \\
\text { metastases }\end{array}$ & 5,902 & 21,884 & Yes & Yes \\
\hline $\begin{array}{l}\text { Giustacchini et al. Nature } \\
\text { Med } 2017\end{array}$ & $\begin{array}{l}\text { Chronic myeloid } \\
\text { leukemia }\end{array}$ & SMART-seq2 & $\begin{array}{l}20 \text { human bone } \\
\text { marrow aspirates }\end{array}$ & 2,287 & 23,384 & No & No \\
\hline
\end{tabular}




\begin{tabular}{|c|c|c|c|c|c|c|c|}
\hline Dataset & Cancer type & $\begin{array}{l}\text { Sequencing } \\
\text { Technology }\end{array}$ & Number of tumors & $\begin{array}{l}\text { Number } \\
\text { of cells }\end{array}$ & $\begin{array}{l}\text { Number } \\
\text { of genes }\end{array}$ & $\begin{array}{l}\text { Annotation } \\
\text { available? }\end{array}$ & $\begin{array}{l}\text { Gene } \\
\text { signature } \\
\text { available? }\end{array}$ \\
\hline Filbin et al. Science 2018 & $\begin{array}{l}\text { H3K27-mutant } \\
\text { glioma }\end{array}$ & SMART-seq2 & $\begin{array}{l}6 \text { human primary } \\
\text { H3K27M-glioma } \\
\text { tumors }\end{array}$ & 4,059 & 23,686 & Yes & No \\
\hline $\begin{array}{l}\text { Jerby-Arnon et al. Cell } \\
2018\end{array}$ & Melanoma & SMART-seq2 & $\begin{array}{l}33 \text { human melanoma } \\
\text { tumors }\end{array}$ & 7,186 & 23,686 & Yes & Yes \\
\hline VanGalen et al. Cell 2019 & $\begin{array}{l}\text { Acute myeloid } \\
\text { leukemia }\end{array}$ & Seq-well & $\begin{array}{l}40 \text { human bone } \\
\text { marrow aspirates }\end{array}$ & 38,410 & 27,899 & No & No \\
\hline $\begin{array}{l}\text { Ting et al. Cell Reports } \\
2014\end{array}$ & $\begin{array}{l}\text { Pancreatic } \\
\text { circulating tumor } \\
\text { cells }\end{array}$ & Tang protocol & $\begin{array}{l}5 \text { mice with pancreatic } \\
\text { cancer, } 1 \text { mouse } \\
\text { embryonic fibroblast } \\
\text { cell line, } 1 \text { mouse } \\
\text { pancreatic cancer cell } \\
\text { line, } 1 \text { control mouse }\end{array}$ & 187 & 29,018 & No & No \\
\hline $\begin{array}{l}\text { Miyamoto et al. Science } \\
2015\end{array}$ & $\begin{array}{l}\text { Prostate circulating } \\
\text { tumor cells }\end{array}$ & ABI SOLiD & $\begin{array}{l}18 \text { patients with } \\
\text { metastatic prostate } \\
\text { cancer, } 4 \text { patients with } \\
\text { localized prostate } \\
\text { cancer, } 12 \text { bulk } \\
\text { primary prostate } \\
\text { tumors, } 4 \text { prostate } \\
\text { cancer cell lines }\end{array}$ & 169 & 21,696 & No & No \\
\hline Jordan et al. Nature 2016 & $\begin{array}{l}\text { Breast circulating } \\
\text { tumor cells }\end{array}$ & SMARTer & $\begin{array}{l}2 \text { ER+/HER2- breast } \\
\text { cancer patients, } 14 \\
\text { triple negative breast } \\
\text { cancer patients }\end{array}$ & 74 & 23,368 & No & No \\
\hline Azizi et al. Cell 2018 & Breast carcinoma & InDrop & $\begin{array}{l}8 \text { human breast } \\
\text { carcinomas }\end{array}$ & 46,016 & 14,875 & No & No \\
\hline
\end{tabular}




\begin{tabular}{|c|c|c|c|c|c|c|c|}
\hline Dataset & Cancer type & $\begin{array}{l}\text { Sequencing } \\
\text { Technology }\end{array}$ & Number of tumors & $\begin{array}{l}\text { Number } \\
\text { of cells }\end{array}$ & $\begin{array}{l}\text { Number } \\
\text { of genes }\end{array}$ & $\begin{array}{l}\text { Annotation } \\
\text { available? }\end{array}$ & $\begin{array}{l}\text { Gene } \\
\text { signature } \\
\text { available? }\end{array}$ \\
\hline $\begin{array}{l}\text { Lambrechts et al. Nature } \\
\text { Med } 2018\end{array}$ & $\begin{array}{l}\text { Non-small cell lung } \\
\text { carcinoma }\end{array}$ & $\begin{array}{l}10 \mathrm{x} \\
\text { Genomics }\end{array}$ & $\begin{array}{l}5 \text { human non- } \\
\text { metastatic lung } \\
\text { squamous carcinoma } \\
\text { tumors }\end{array}$ & 51,775 & 22,180 & Yes & Yes \\
\hline $\begin{array}{l}\text { Davidson et al. bioRxiv } \\
2018\end{array}$ & Melanoma & SMART-seq2 & ? mouse tumors & 6,423 & 26,946 & No & No \\
\hline $\begin{array}{l}\text { Peng et al. Cell Research } \\
2019\end{array}$ & $\begin{array}{l}\text { Pancreatic ductal } \\
\text { adenocarcinoma }\end{array}$ & $\begin{array}{l}10 \mathrm{x} \\
\text { Genomics }\end{array}$ & $\begin{array}{l}24 \text { human primary } \\
\text { pancreatic ductal } \\
\text { adenocarcinoma } \\
\text { tumors, } 11 \text { control } \\
\text { pancreases }\end{array}$ & 57,530 & 24,005 & Yes & Yes \\
\hline $\begin{array}{l}\text { Darmanis et al. Cell } \\
\text { Reports } 2017\end{array}$ & $\begin{array}{l}\text { Primary } \\
\text { glioblastoma }\end{array}$ & SMART-seq2 & $\begin{array}{l}4 \text { human glioblastoma } \\
\text { tumors }\end{array}$ & 3,589 & 23,465 & No & No \\
\hline $\begin{array}{l}\text { Kumar et al. Cell } \\
\text { Reports } 2018\end{array}$ & $\begin{array}{l}\text { Mixed cancer: } \\
\text { Melanoma, breast } \\
\text { mammary } \\
\text { carcinoma, Lewis } \\
\text { lung carcinoma, } \\
\text { colon carcinoma, } \\
\text { fibrosarcoma }\end{array}$ & $\begin{array}{l}10 \mathrm{x} \\
\text { Genomics }\end{array}$ & $\begin{array}{l}1 \text { mouse melanoma } \\
\text { tumour, } 1 \text { mouse breast } \\
\text { mammary carcinoma } \\
\text { tumour, } 1 \text { mouse } \\
\text { Lewis lung carcinoma } \\
\text { tumor, } 2 \text { different } \\
\text { mouse colon } \\
\text { carcinoma tumors, } 1 \\
\text { mouse fibrosarcoma } \\
\text { tumor }\end{array}$ & 10,573 & 27,998 & No & No \\
\hline $\begin{array}{l}\text { Zhao et al. BMC Med } \\
\text { Genomics } 2019\end{array}$ & $\begin{array}{l}\text { Glioblastoma cell } \\
\text { line }\end{array}$ & Fluidigm C1 & $\begin{array}{l}1 \text { human glioblastoma } \\
\text { cancer cell line, } 1 \\
\text { normal neural stem } \\
\text { cell line }\end{array}$ & 134 & 21,209 & No & No \\
\hline
\end{tabular}

